\title{
Encéphalopathie associée au métronidazole : le signe de la " châtaigne »
}

\section{Metronidazole Associated-Encephalopathy: Chestnut Sign}

\section{A. Elkoundi · M. Rabii Andaloussi · M. Bensghir}

Reçu le 22 octobre 2017; accepté le 7 décembre 2017

(C) SFMU et Lavoisier SAS 2017
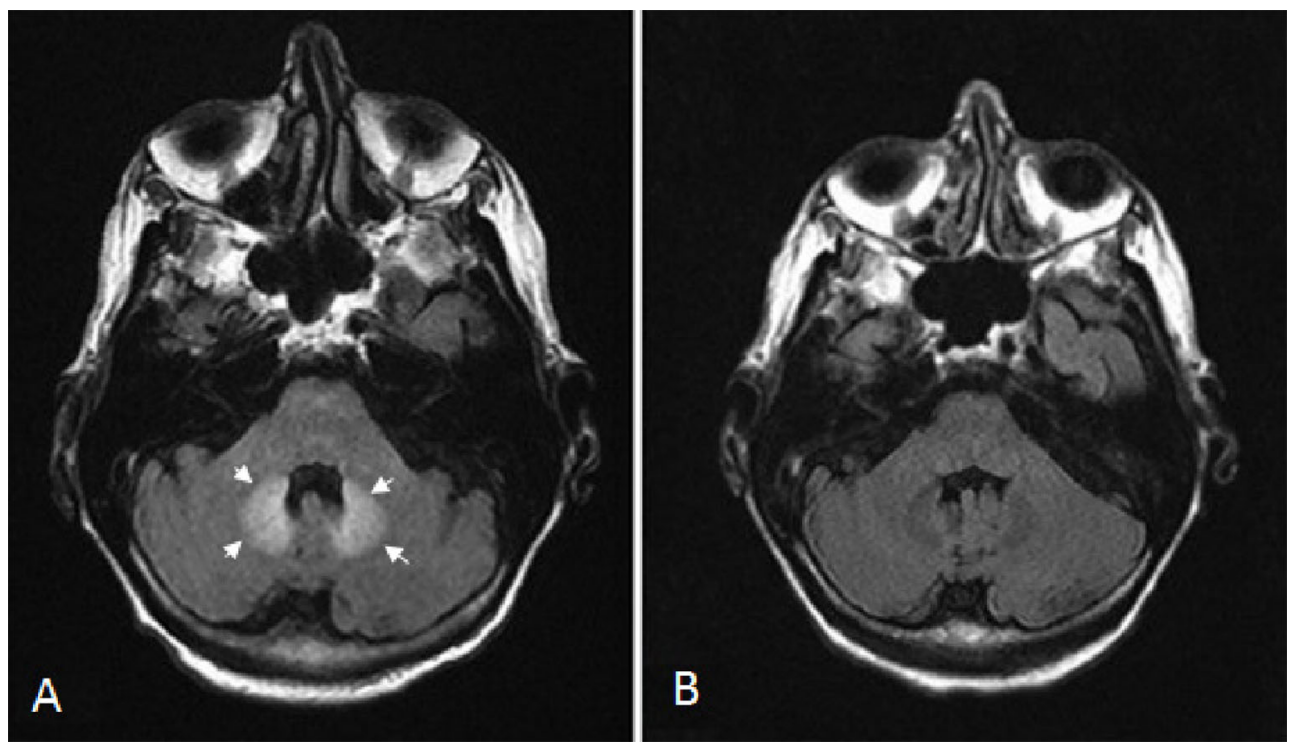

Fig. 1 IRM cérébrale en coupe axiale séquence FLAIR montrant : A/ signe de la châtaigne sous forme d'un hypersignal bilatéral et symétrique des noyaux dentelés (flèches en blanc) ; B/ disparition du signe après arrêt du métronidazole

Une femme âgée de 59 ans est admise aux urgences pour confusion mentale après une chute à son domicile, précédée durant quelques jours d'une dysarthrie et d'une ataxie cérébelleuse dans un contexte d'apyrexie. Deux semaines auparavant, elle avait été opérée pour une fistule anale pour laquelle elle avait été mise sous antibiothérapie à base de métronidazole $500 \mathrm{mg}$ deux comprimés, trois fois par jour. Le bilan biologique ne montre pas de signes de malabsorption. Une anémie macrocytaire par carence en folates ou en vitamine B12 est absente et les taux sanguins de vitamine B1 et $\mathrm{E}$ sont physiologiques. Le scanner cérébral et le LCR sont strictement normaux. L'IRM cérébrale en séquence FLAIR révèle des hypersignaux bilatéraux et symétriques des noyaux dentelés cérébelleux (Fig. 1A). L'arrêt du métro-

\footnotetext{
A. Elkoundi $(\square) \cdot$ M. Rabii Andaloussi $\cdot$ M. Bensghir Département d'anesthésie et réanimation de l'hôpital militaire d'instruction Mohamed V, Faculté de médecine et de pharmacie de Rabat, université Mohamed V, 10100 Rabat, Maroc e-mail : abd.el.koundi@gmail.com
}

nidazole a permis une amélioration clinique et également radiologique progressive (Fig. 1B).

Le métronidazole peut entrainer plus habituellement une neuropathie périphérique et une leuconeutropénie. L'encéphalopathie induite au métronidazole est exceptionnelle mais grave. Bien que non encore élucidé, il semble que le mécanisme physiopathologique soit lié à un trouble du métabolisme de la thiamine [1]. L'aspect IRM est caractéristique, avec des anomalies du signal de topographie assez typique en forme de « châtaigne » [2], habituellement réversible à l'arrêt précoce du traitement.

\section{Références}

1. Alston TA, Abeles RH (1987) Enzymatic conversion of the antibiotic metronidazole to an analog of thiamine. Arch Biochem Biophys 257:357-62

2. Kuriyama A (2017) Chestnut sign: metronidazole-induced encephalopathy. J Emerg Med 52:101-2 